\title{
High efficiency operation by water temperature and area control in thermal radiative cooling/heating system
}

\author{
Hiroaki Ikegami $^{\mathrm{a}^{*}}$, Akihiro Satake ${ }^{\mathrm{a}}$, Hisafumi Yamada ${ }^{\mathrm{a}}$, Yasunori Mitani ${ }^{\mathrm{a}}$ \\ ${ }^{a}$ Department of Electrical and Electronic Engineering, Kyushu Institute of Technology, Kitakyushu-shi, Fukuoka, 804-8550, Japan
}

\begin{abstract}
In recent years, energy conservation measure in the demand side has been promoted for energy problems. Among these the cooling/heating system accounts for about a quarter of the energy consumption in the home of Japan, the energy conservation measures of the cooling/heating system are particularly important. Research on energy savings and performance evaluation in thermal radiative cooling/heating system is carried out. In this paper, this system is verified about the method of heating operation with high energy efficiency. As a method, heat discharge is controlled by operating water temperature and passing water area of radiator which is emulated by the forced heat exchange with a fan in heating operation. As a result, lowering water temperature and increasing passing water area gets high energy efficiency with getting the same heat discharge.
\end{abstract}

Keywords: Thermal radiative cooling/heating system, heat pump, energy saving, radiant heat transfer

\section{Introduction}

In the recent years, energy consumption around the world is continuously increasing and the depletion of fossil fuel along with problems concerning global warming is becoming more severe [1]. As a result, there is a growing concern in energy conservation countermeasures applicable in the demand side. As energy-saving measures, several studies have been carried out. For example, visualization of power and wall greening have been reported to be an effective mean of energy conservation [2], [3]. This research focuses on heat and cooling systems which accounts for a quarter of the total energy consumption in the consumer of Japan [4]. We have studied the performance energy conservation of the thermal radiative cooling/heating system with radiant heat transfer concept and heat pump [5]-[8]. The heat pump is the equipment with high efficiency because it is possible to obtain the thermal energy several times more than the power used [9].

The thermal radiative cooling/heating system is constructed with interior radiators and exterior unit of a heat-exchanger unit and heat pump. Fig. 1 shows photograph of the aluminum radiator. In addition, Fig. 2 shows the block diagram of this system. During heating, the heat in the outside air is absorbed through the exterior unit, the water is then heated, and the heat is transferred inside the room through the circulation of the water between the heat-exchanger and the interior radiator. In transferring heat to the room, the heating and cooling mechanism is achieved by actively using the radiant heat transfer through the far-infrared rays from the radiator [7]. The ceramic material coating with high emissivity far-infrared region was applied to the walls, ceiling, and radiator surface to increase the radiation heat transfer effect. Moreover, the system characteristics include non-emission of warm and cold air flows due to the use of radiant heat transfer, ability to maintain a clean environment without flinging up the dust, low heat variations due to the use of circulating water in the radiator, and non-emission of machine noise [10]. During cooling, a dehumidification effect through the condensation at the surface of radiator maintains the comfortable indoor environment.

\footnotetext{
* Manuscript received June 15, 2014; revised July 30, 2014.

Corresponding author: Hiroaki Ikegami; Tel.: +81-93-884-3243; E-mail address: j108005h@ mail.kyutech.jp.

doi: $10.12720 /$ sgce.3.4.437-440
} 


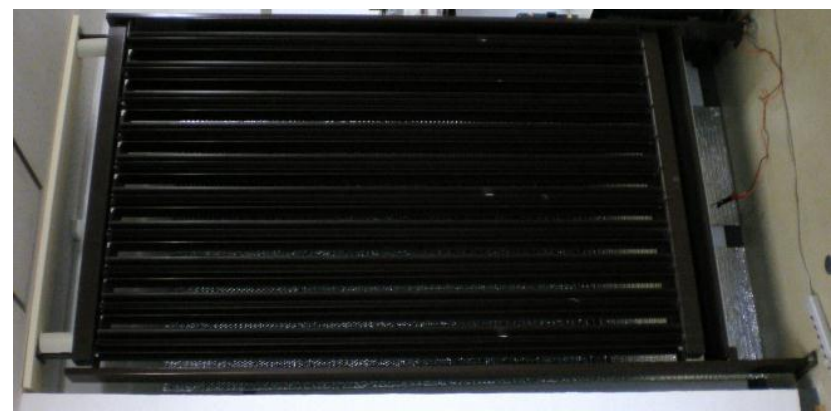

Fig. 1. Photograph of the aluminum radiator.

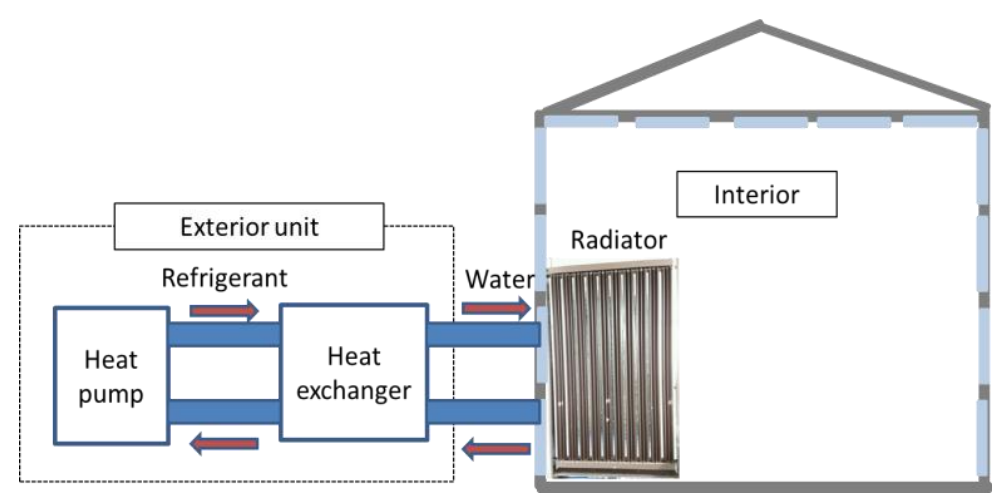

Fig. 2. Block diagram of the thermal radiative cooling/heating system.

In this paper, the operation with high energy conservation in thermal radiative cooling/heating system was investigated by coefficient of performance (COP) in heating that obtained through emulating experiment. As a method, heat discharge was controlled by variation of passing water area and water temperature. Furthermore, COP was calculated by diving heat discharge by power consumption.

\section{Experimental COP Evaluation by Emulating the Increase of Passing Water Area}

\subsection{Experimental background}

In the experiment, the thermal radiative cooling/heating system with an indoor aluminium radiator was used, which is installed in a room with a floor area of $50 \mathrm{~m}^{2}$ and ceiling height of $2.6 \mathrm{~m}$ on the $4^{\text {th }}$ floor of a 4-story building inside Kyushu Institute of Technology Tobata campus. In this system, the heat discharge is controlled mainly by the regulation of the flowing water temperature in the radiator. Also, with the same water temperature settings, it is observed that lowering the outflowing water temperature to the heat exchanger increases COP. In this case, when the temperature difference between the inflowing and outflowing water in the radiator is increased, COP also increases [8]. Moreover, it is easily guessed that COP is improved through the lowering of the outflowing water temperature by increasing the passing water area. However, it is structurally impossible to vary the area of the current radiator under study. To emulate the same effect of increasing the area of the radiator, an electric fan with setting of low, medium, and high air flows is used to lower the temperature of the outflowing water. Here, the relationship of the equivalent area emulated by the air flows is observed. Through the management of the water temperature and passing water area, the heat discharge is controlled for high COP.

\subsection{Verification of emulated passing water area by air flow}

First, the passing water area emulated by air flows is verified. There are 10 aluminum radiators and water flows in each of these radiators (as seen on Fig. 1). Therefore, assuming that the heat discharge is proportional to difference of temperature between water temperature $T$ and room temperature $T_{\text {room, }}$ and 
letting the number of radiators be $x$, and the inflowing water temperature be $T_{0}$, the water temperature $T$ in the $x$ th radiator can be expressed in the following exponential equation as:

$$
T=\left(T_{0}-T_{\text {room }}\right) \exp (-\alpha x)+T_{\text {room }}
$$

Under room temperature of $21^{\circ} \mathrm{C}$, measuring the temperature of the inflowing water in each of the radiators and fitting of the data to satisfy in equation (1) resulted to $\alpha=0.011$ in each radiator. Given the inflowing and the outflowing water temperature and by using equation (1), the number of radiators corresponding to the amount of air flow is estimated. In low air flow, the number of radiators is estimated to be 15 . In medium and in strong air flow, it is estimated to be 17 and 19, respectively. In the case of 10 radiators, the passing water area is $3.6 \mathrm{~m}^{2}$. In low, medium, and high air flow, the passing water area corresponding to $5.4,6.1$, and $6.9 \mathrm{~m}^{2}$ were determined.

\subsection{Evaluation of heat discharge and change of temperature of the outflowing water}

The heat discharge and the change in temperature of the outflowing water corresponding to a change in area emulated by different air flows are shown in Fig. 3 and Fig. 4. In each temperature setting, it is observed that the heat discharge is increased and the outflowing water temperature is lowered by increasing the passing water area (air flow). It is found that a maximum of $40 \%$ increase in the heat discharge is achieved by variation of the passing water area, and the percentage rise is increased when the temperature setting is also increased. Also, a same heat discharge is obtained with a passing water area of $6.9 \mathrm{~m}^{2}$ at $40^{\circ} \mathrm{C}$ and with a passing water area of $3.6 \mathrm{~m}^{2}$ at $50^{\circ} \mathrm{C}$. Moreover, an equal heat discharge can be obtained by increasing the passing water area even in the case of low or higher water temperature.

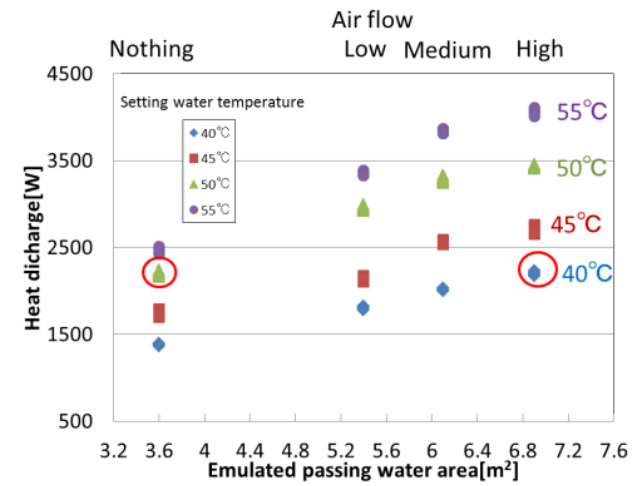

Fig. 3. Variation of heat discharge by passing water area.

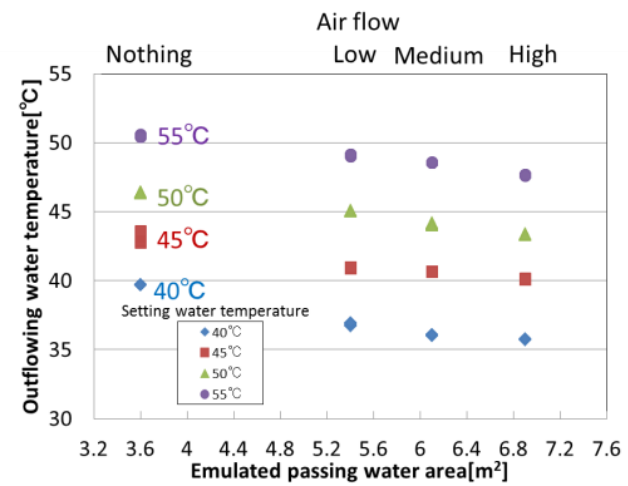

Fig. 4. Variation of outflowing water temperature by passing water area.

\subsection{Evaluation of energy conservation through passing water area and water temperature control}

Fig. 5 shows the variation of COP corresponding to changes in the passing water area in $40^{\circ} \mathrm{C}$ and $50^{\circ} \mathrm{C}$ water temperature. In the same temperature settings, COP is improved and is found to be proportional to the passing water area. In Fig. 5, the red circle corresponds to a passing water area of $6.9 \mathrm{~m}^{2}$ at $40^{\circ} \mathrm{C}$, and the passing water area of $3.6 \mathrm{~m}^{2}$ at $50^{\circ} \mathrm{C}$ in which both cases has $2200 \mathrm{~W}$ heat discharge (as seen on Fig. 3). The power consumption is $670 \mathrm{~W}$ in the case of $40^{\circ} \mathrm{C}$, and $880 \mathrm{~W}$ in the case of $50^{\circ} \mathrm{C}$. Thus, with the same heat discharge, power consumption is $24 \%$ lower in the case of $40^{\circ} \mathrm{C}$. Also, the temperature of the outflowing water in the case of 40 , and $50^{\circ} \mathrm{C}$ are 35.7 and $46.4^{\circ} \mathrm{C}$, respectively. Accordingly, the COPs in two cases are 3.3 and 2.5. The reason that increase of COP is presumed because outflowing water temperature lowered by lowering of setting water temperature and increasing of passing water area. Fig. 6 is a graph. Its horizontal axis is the heat discharge when varying emulated passing water area and setting water temperature, and vertical axis is COP. When getting same amount of heat discharge, COP tends to be high so that setting water temperature is low. That is, in the case of comparable heat discharge, COP is high by lowering of setting water temperature and increasing of passing water area in heating operation. 


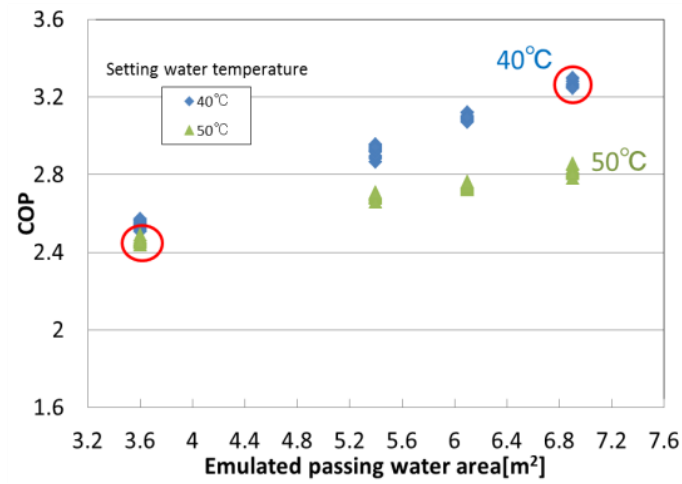

Fig. 5. Variation of COP by passing water area.

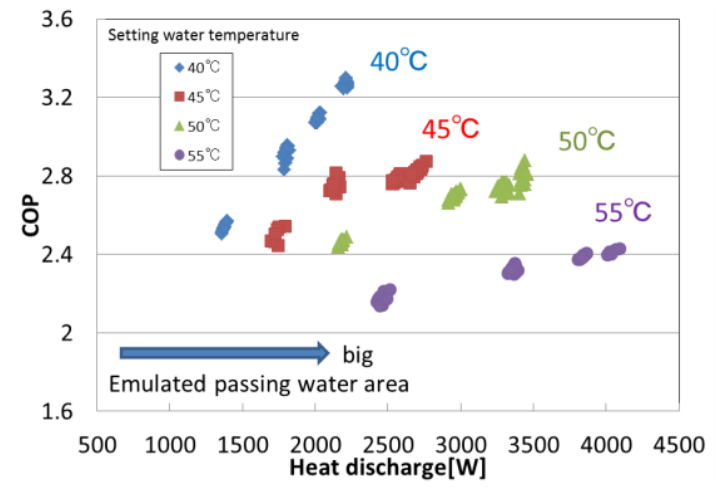

Fig. 6. Graph of heat discharge and COP.

\section{Conclusions}

The study on controlling of the heat discharge by the variation of the passing water area and the setting water temperature using a thermal radiative cooling/heating system for a high-efficiency heating operation is conducted. As a result, it is found that heat discharge varied by changing the passing water area, other than changing the setting water temperature. Furthermore, it is found that high energy efficiency is obtained by lowering water temperature and increasing passing water area with getting the same heat discharge. In future, it is necessary to verify the efficiency by performing the same operation in the cooling operation. A more efficient energy saving operation can be expected by adding new control elements in conventional operations.

\section{References}

[1] Yamada N, Utiya E, Konno Y. Schematic-Basis of Air Conditioning. Natsume CO., LTD; 2009:88.

[2] Nozaki Y, Mitani Y, Qudaih Y, Nanano G, Fujita T. Simple measurement system for indoor power flow distributions using voltmeters at electrical outlet. In: Proc. The International Conference on Electrical Engineering, 2011:1-1.

[3] Nishio Y, Mitani Y, Ito K, Soejima Y. Quantitative evaluation for the wall greening based on reduction of air conditioners power consumption and green plant energy production. In: Proc. The International Conference on Electrical Engineering, 2009:ICEE-A182.

[4] Agency for Natural Resources and Energy of Japan. Energy White Paper. WITH UP CO., LTD; 2013:108.

[5] Murakami K, Yamada H, Mitani Y, Miyamoto N, Futaeda T. Energy saving and power management using thermal radiative cooling/heating system. In: Proc. National Convention by Institute of Electrical Engineers of Japan, 2011:I-5.

[6] Ikegami H, Sato H, Satake A, Yamada H, Mitani Y, Miyamoto N, Futaeda T. Radiator water temperature dependence of cooling and heating coefficient of performance in thermal radiative cooling/heating system. In: Proc. Kyushu Section Convention by Institute of Electrical Engineers in Japan, 2013: 06-1P-08.

[7] Sato H, Satake A, Yamada H, Mitani Y, Miyamoto N, Futaeda T. Power suppression operation by thermal radiative cooling/heating system with effective radiant heat transfer. In: Proc. National Convention by Institute of Electrical Installation Engineers of Japan, 2013:B-8.

[8] Satake A, Sato H, Ikegami H, Yamada H, Mitani Y, Miyamoto N, Futaeda T. Power suppression operation using temperature and flow control in thermal radiative cooling/heating system. In: Proc. National Convention by Institute of Electrical Engineers of Japan, 2014:4-185.

[9] Tanaka S, Yatabe T. Schematic-Heat Pump. Ohmsha CO., LTD; 1995:21.

[10] Japanese Association of Bulding Mechanical and Electrical Engineers. Working Knowledge of Air Conditioning and Hot Water Supply System with Heat Pump for Designer. Ohmsha CO., LTD; 2010:12-13. 